\title{
Designing Conversational Interfaces to Reduce Dissonance
}

\section{Meira Chefitz}

University of Michigan

Ann Arbor, MI 48109, USA

meira@umich.edu University of Michigan

Ann Arbor, MI 48109, USA jausbren@umich.edu

\section{Nigel Melville}

Ann Arbor, MI 48109, USA

npmelv@umich.edu

\section{Jesse Austin-Breneman}

University of Michigan

\begin{abstract}
Conversational interfaces-computer interfaces that use text or voice for human-computer interaction-are one of many interaction modalities in interactive systems. Their use has expanded with the growth and range of products that are both digital and physical in nature. But users' expectations of conversational experiences are not being met. In addition, expectations may not be met in different ways across people.

This paper aims to provoke dialogue among design researchers and practitioners regarding the design of an interaction modality that is commonly found in human-to-human interaction. It focuses on the design of conversational interfaces such as virtual assistants to illustrate the quandary. Finally, the paper proposes a

phenomenon for the issue-a type of dissonance-and introduces tactics to reduce dissonance. It sheds light on potential approaches to design as well as complications that may occur.
\end{abstract}

\section{Author Keywords}

Conversational interfaces; interaction design strategy personal or classroom use is granted without fee provided that copies are not made or distributed for profit or commercial advantage and that copies bear this notice and the full citation on the first page. Copyrights for third-party components of this work must be honored. For all other uses, contact the Owner/Author.

DIS'18 Companion, June 9-13, 2018, Hong Kong

(c) 2018 Copyright is held by the owner/author(s).

https://doi.org/10.1145/3197391.3205439

\section{ACM Classification Keywords}

H.5.m. Information interfaces and presentation (e.g. $\mathrm{HCI}$ ): Miscellaneous

\section{Introduction}

Conversational interfaces are human computer interfaces through which humans and computers

interact in conversation. Recently, they have been 
designed into a variety of systems and products including chatbots, voice activation systems and virtual assistants. In the decades that conversational

interfaces have been designed, there has been an effort to design them to be humanlike. Today, Amazon's virtual assistant Alexa can laugh and apologize. The benefits and controversy in the use of anthropomorphism in interaction design is known [1] There is a business goal behind this in which humanlikeness increases users' pleasure in the experience as well as level of trust $[1,2]$. Although anthropomorphism can be applied to design to meet business goals, the design decisions, implementation, and execution could disappoint or trouble users [1].

The downsides to designing for human-likeness extend beyond poor implementation or selecting inappropriate factors for anthropomorphism. The way designers choose to measure success impacts how designers choose to design. Usability measures of preference, for example, have resulted in inflated reports in favor of human-likeness in the form of human voice over machine-synthesized speech [3]. At this point, it is recognized that design strategies should be more sophisticated than to simply design for humanlikeness-that context and situations matter [4]. For instance, matching a user's emotional state in conversational interaction while driving a car can lead to better driving performance by the user [5]. In contrast is the design of a genderless chatbot in which a factor associated with human-likeness is intentionally omitted from the design [6]. The field has reached a milestone in product maturity in that designing a particular conversational interface to lack human characteristics can be considered a market differentiator.

\section{Unmet expectations: the conversational}

\section{interface problem}

While conversational interfaces continue to evolve, they have not reached a pinnacle of design. Recent developments in the design of conversational interfaces point to the unmet expectations of users in interaction with conversational interfaces. For instance, Amazon has worked with mental health experts to modify responses to queries on well-being after criticism from medical researchers on performance $[7,8]$. In this approach, the solution of situational and contextual design is to consult with domain experts who have an understanding of how to interact in human conversation rather than use known research methods such as design ethnography [9].

While relying on domain experts in human-human interaction to inform the design of human computer interaction might appear intuitive, it feeds into a system of striving for human-likeness in all forms of interaction. It omits the design and interaction strategy from the equation. In turn, design practitioners are trained to take a single approach. This approach assumes that voice interfaces should behave as humans do, but in ways that have not been proven.

Designing with domain experts' knowledge of human interaction does not address variations in unmet expectations. A fundamental problem in conversational interfaces is that we are taking an inherently human interaction and transforming it for human computer interaction. While it might seem like conversational experiences are the easiest to design for, this implied naturalness exposes the responsibilities and struggles imparted on designers. Domain experts know domains, but the approach is generalized across users. Users are not interacting with people in conversational interfaces; they are interacting with machines. We are still learning what that means as designers, and users are still learning what to expect. This learning process is exposing human differences in what people expect and how salient those attitudes are.

\section{Theoretical mechanism underlying unmet}

\section{needs: cognitive dissonance}

The study of dissonance may illustrate the design challenges when addressing unmet expectations. 
Cognitive dissonance is a theory that describes the tension an individual may experience when holding two elements of cognition-such as a fact or belief-that oppose each other [10]. It theorizes that individuals want their beliefs and actions to be consistent. When cognitive elements do not fit together, they are not consistent leading to dissonance [11]. Dissonance can be experienced at varying degrees of intensity depending on the cognitions [10]. Dissonance can explain our unmet expectations when interacting with a conversational interface. In a layering of theories, cognitive dissonance has been proposed to be a contributor to the uncanny valley on the individual user level [12].

Only relatively recently have we been able to use conversation to interact with machines and we are still forming beliefs and opinions about how conversational interaction should perform. We are doing it at different paces and with different elements collectively, leading to a variety of dissonance across people-a diversity of unmet expectations (Figure 1).

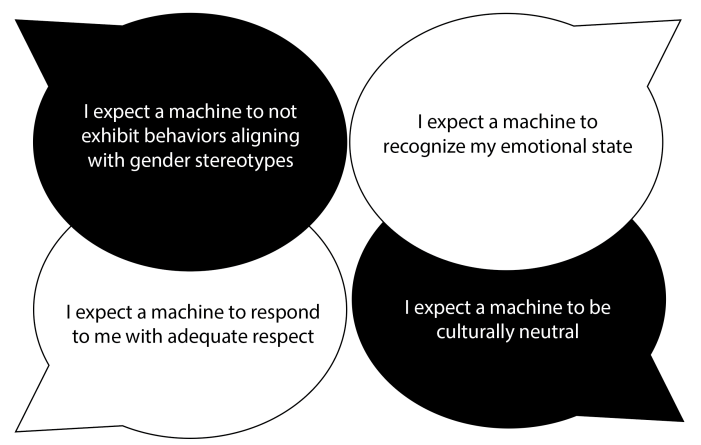

Figure 1: Users have expectations of interaction with humans and expectations of interaction with machines. Both may be held in the interaction with conversational interfaces. These may conflict and they may vary across people. The visual summarizes evaluations of conversational interfaces from two separate sources $[7,13]$.

Communication is collective but can also be deeply segmented and individualized. The varying expectations that people have of natural conversation and of

machine identity leads to diversity in dissonance. This is a challenging design problem because it is specific to contexts and situations as well as the individual. Segmentation can be inconsistent. In a study testing user preference of a personal assistant on a shopping website, some anthropomorphic details were perceived more useful than others based on segments of users [14].

\section{Reducing dissonance}

If people experience dissonance-albeit in different ways-could the reduction of dissonance be a viable approach to the design of conversational interfaces?

Generally, dissonance is reduced when an attitude or behavior changes $[10,11]$. Individuals may shift their subsequent behaviors, or they can shift their attitudes about an experience or concept. The amount or level of dissonance that one can experience is determined by a ratio of dissonant cognitions to non-dissonant cognitions [10]. If an interactive system does not entail forced compliance [15], cognitive dissonance may be identified by behavioral changes by the user.

Reducing dissonance through behavior and attitude Two simple techniques to reduce users' dissonance attempt to adjust a user's behavior or attitude directly after an interaction, particularly when the level of dissonance is low. One option is to do nothing. Allow the users to learn from the experience of unmet expectations so that they may shift their behavior in interaction in the future. Another option is to provide cues to the users on why expectations may not have been met, sharing the interface's intent and expected behavior. This could alter users' expectations and 
therefore general attitude regarding interaction with the conversational interface.

A challenge in the approach is in generalizing when dissonance occurs. Dissonance is tied to one's beliefs and actions. These can be shared across people but they are not universal. Research in dissonance has found differences based on group values and cultural rules, as well as on gender and class [15]. While there is a great depth and breadth of research in dissonance that enables generalization of results, dissonance occurs in different strengths across the known factors.

Reducing dissonance through avoidance Individuals may reduce dissonance by avoiding situations that induce it [10]. Designers may find ways to encourage use of a conversational interfaces while also dissuading users when the domain, situation, context or topic is out of scope. Imagine that a person sends a query to a conversational interface that the system is not designed to engage in. The outcome might be increased dissonance in the user, but it is also a learning experience for future engagement. In this case, reminders of the scope and nature of the assistant throughout the experience could benefit users in continued use.

Reducing dissonance through choice

Similar to shaping users' attitudes during or after an interaction, designers could choose to set expectations in advance. In a study on dissonance, participants who chose to work with a partner on a game and were aware of the partner's limitations in advance may have experienced a higher level of dissonance, but they also liked the partner more than if they hadn't been told the limitations in advance [15]. Applied to the design of conversational interfaces, exposing scope and

boundaries of conversational interaction in advance and associating those with users' choices might improve the user experience.
The ability to make a choice about one's actions reduces dissonance [15]. As users are still becoming accustomed to conversational interfaces, providing them with optional modalities may improve the experience. The design of interactive systems play a significant role here.

Context specificity and personalization in interface design suggest further design research into AI-driven conversational interfaces. This could allow designers to identify when dissonance occurs or design parameters for which customization may reduce dissonance.

\section{Conclusion}

People have expectations that are not being met when interacting with conversational interfaces. Those expectations vary. The simplest answer to addressing diversity in those expectations is to personalize the experiences-to design for individuals and not the collective. However, the implications of successfu personalized experiences based on simple

segmentation or patterned generalization may outweigh the current unmet expectations. Furthermore personalized experiences add another level of social responsibility to the design strategy. Another solution would be to strategically apply interactive approaches to the reduction of dissonance when it has been identified through user behavior.

\section{Acknowledgements}

This material is based upon work supported by the National Science Foundation Graduate Research Fellowship under Grant No. DGE 1256260. Any opinions, findings, and conclusions or recommendations expressed in this material are those of the authors and do not necessarily reflect the views of the sponsors.

\section{References}

1. Yvonne Rogers, Helen Sharp, and Jenny Preece. 2011. Interaction Design: Beyond HumanComputer Interaction. John Wiley \& Sons, 2011. 
2. Lingyun Qiu and Isak Banbasat. 2010. Online Consumer Trust and Live Help Interfaces: The Effects of Text-to-Speech Voice and ThreeDimensional Avatars. International Journal of Human-Computer Interaction. 19:1. 75-94. DOI: https://doi.org/10.1207/s15327590ijhc1901_6

3. Wade J. Mitchell, Chin-Chang Ho, Himalaya Patel, and Karl F. MacDorman. 2011. Does social desirability bias favor humans? Explicit-implicit evaluations of synthesized speech support a new $\mathrm{HCI}$ model of impression management. In

Computers in Human Behavior. 27. 402-412. DOI: https://doi.org/10.1016/j.chb.2010.09.002

4. Selma Sabanovic, Marek P. Michalowski, and Reid Simmons. 2006. Robots in the wild: Observing human-robot social interaction outside the lab. In International Workshop on Advanced Motion Control, AMC, 2006, 576-581. DOI:https://doi.org/10.1109/AMC.2006.1631758

5. Clifford Nass and Scott Brave. 2005. Wired for speech: How voice activates and advances the human-computer relationship. MIT press, Cambridge.

6. Madeline Buxton. 2017. How You Speak to Siri and Alexa Matters More Than You Think-Here's Why. (March 2017). Retrieved March 12, 2018 from https://www.refinery29.com/2016/11/126073/kasi sto-kai-bot-ai-sexism

7. Aaron P. Calvin. 2017. Can Amazon's Alexa Be Your Friend? (March 2017). Retrieved March 12, 2018 from https://digg.com/2017/amazon-alexa-is-notyour-friend

8. Adam S. Miner, Arnold Milstein, Stephen Schueller, Roshini Hegde, Christina Mangurian, and Eleni Linos. 2016. Smartphone-Based Conversational Agents and Responses to Questions About Mental Health, Interpersonal Violence, and Physical Health. JAMA Internal Medicine. 311(18), 1851-1852.
DOI:https://doi.org/10.1001/jamainternmed.2016. 0400

9. $\quad$ Nigel P. Melville and Michael Hopps. 2012. Systematic Service Innovation in Organizations: EDCI--A Template for Human-Centered Design of E-Services. In 2012 45th Hawaii International Conference on System Sciences. 4112-4118. DOI:https://doi.org/10.1109/HICSS.2012.37

10. Jack W. Brehm and Arthur R. Cohen. 1962. Explorations in Cognitive Dissonance. Wiley, NY, NY.

11. Leon Festinger. 1957. A theory of Cognitive Dissonance. Row, Peterson, and Company, Evanston, IL.

12. Anne E. Ferrey, Tyler J. Burleigh, and Mark J. Fenske. 2015. Stimulus-category competition, inhibition, and affective devaluation: a novel account of the uncanny valley. Frontiers in Psychology. 6, Article 249 (March 13, 2015), 15 pages. DOI

https://doi.org/10.3389/fpsyg.2015.00249

13. Leah Fessler. 2017. We Tests Bots Like Siri and Alexa To See Who Would Stand Up to Sexual Harassment. Retrieved April 5, 2018 from https://qz.com/911681/we-tested-apples-siriamazon-echos-alexa-microsofts-cortana-andgoogles-google-home-to-see-which-personalassistant-bots-stand-up-for-themselves-in-theface-of-sexual-harassment/

14. Lingyun Qiu and Isak Banbasat. 2010. A study of demographic embodiments of product

recommendation agents in electronic commerce. In International Journal of Human-Computer Studies. 68. 669-688. DOI: https://doi.org/10.1016/j.ijhcs.2010.05.005

15. Joel Cooper. 2007. Cognitive Dissonance: Fifty Years of a Classic Theory. Sage, London. 